\title{
ARAÇ İÇI SESİNDEN ARACI TANIMA VE SINIFLANDIRMA
}

\author{
${ }^{1}$ Emrah AYDEMIR (D) , ${ }^{2}$ Murat IŞIK \\ 1,2Ahi Evran Üniversitesi, Mühendislik-Mimarlık Fakültesi, Bilgisayar Mühendisliği Bölümü, Kırşehir, TÜRKIYYE \\ ${ }^{1}$ emrah.aydemir@ahievran.edu.tr, ${ }^{2}$ muratisik@ahievran.edu.tr
}

(Geliş/Received: 20.06.2020; Kabul/Accepted in Revised Form: 03.11.2020)

ÖZ: Günümüzde, teknolojik imkanların hızla gelişmesiyle ses sınıflandırma uygulamalarının sayıları da artmakta ve popüler bir çalışma alanı haline gelmektedir. Bu çalışmada, amacımız durağan halde bir aracın üretmiş olduğu sesi kullanarak "aracın sesli imzasını" üretmek ve aracın sınıflandırılması için kullanmaktır. Çalışan bir aracın sesi; motor sesi, titreşimden kaynaklı sesler, rüzgâr sesleri gibi bazı seslerin bir araya gelmesiyle oluşur. Uygulamada 22 aracın rölantideki sesleri kaydedilmiş ve Local Binary Pattern (LBP) ve Cubic SVM algoritmaları kullanılarak \%95,2 oranında başarılı sınıflandırılmıştır. Ayrıca, elde edilen sonuçlar literatürdeki çalışmalarla karşılaştırılmıştır.

Anahtar Kelimeler: ses sınıflandırma, araç tanıma, sesli imza, araç sımflandırma

\section{Vehicle Detection and Classification from Its Interior Sound}

\begin{abstract}
Today, with the rapid development of technological possibilities, the number of sound classification applications are increasing and becoming a popular field for researchers. In this study, our aim is to extract "vehicle sound signature" by using the sound produced by the vehicle at idle mode. After that to use this sound signature for the classification of the vehicle. The sound of a working vehicle at idle mode consist of some noises cause by engine, vibration, wind etc. In practice, the sounds of 22 vehicles at idle mode were recorded and 95.2\% successful classification was made by using the Local Binary Pattern (LBP) method and the Cubic SVM algorithm. In addition, the results were analyzed by comparing them with similar studies in the related literature.
\end{abstract}

Key Words: sound classification, vehicle recognition, voice signature, vehicle classification

\section{GİRİŞ (INTRODUCTION)}

Son yıllarda ses sınıflandırma konusu araştırmacıların giderek daha çok ilgilerini çeken bir çalışma alanı haline gelmiştir (Han ve diğ., 2016; Mielke ve Brück, 2013; Salamon ve diğ., 2014; Salamon ve Bello, 2017). Bunun başlıca nedeni ise günümüz teknolojisi ile ses sinıflandırma yönteminin kullanıldığ1 uygulamaların ve alanların oldukça geniş olmasıdır. Ses sınıflandırma yönteminin kullanıldığı başlıca uygulamalar; hayvan seslerinin sınıflandırılması, konuşma/müzik gibi birbirinden farklı seslerin ayrıştııılması, insan seslerinin sınıflandırılması, ortam seslerinin sınıflandırılması, araç seslerinin sinıflandırılması gibi uygulamalardır (Chu ve diğ., 2008; Huang ve Hansen, 2006; Lavner ve Ruinskiy, 2009; Montino ve Pau, 2019; Scheirer ve Slaney, 1997; Zhang ve Kuo, 2001). Ses sinıflandırma yönteminin kullanıldığı başlıca alanlar ise güvenlik, sağlık, askeri amaçlar, gözetim ve takip sistemleri, akıllı algılama ve kontrol sistemleri, eğlence sistemleri, kentsel uygulamalar ve Akıllı Ulaşım Sistemleri şeklindedir (Cowling ve Sitte, 2003; Dokur ve Ölmez, 2008; Jarnicki ve diğ., 1998; Kubera ve diğ., 2015; Mielke ve Brück, 2013; Radhakrishnan ve diğ., 2005; Salamon ve Bello, 2015; Yoo ve Yook, 2008). Araç tanıma ve 
sınıflandırma konusu, Akıllı Ulaşım Sistemlerinde de karşılaşılan en temel zorluklardan biridir (De Angelis ve diğ., 2016; Tan ve diğ., 2018).

Literatürde bu tür zorluklara çözüm olarak araç kabin sesleri, yol sesi, motor sesini ve lastik sesini veri olarak kullanarak farklı sınıflandırma sonuçlarına ulaşan birçok çalışma mevcuttur (Alexandre ve diğ., 2015; Erb, 2007; George ve diğ., 2013; Ghiurcau ve Rusu, 2009; Johnstone ve Woodward, 2013; Mayvan ve diğ., 2015; Montino ve Pau, 2019; Paulraj ve diğ., 2013; Wieczorkowska ve diğ., 2018). Ancak bu çalışmaların ortak amacı araç türünü tespit etmeye yöneliktir. Bu çalışma ile sunulan yöntemde ise motor sesi kaydedilen bir aracın daha sonradan tespiti hakkında tahminde bulunmaya yöneliktir.

Çalışan bir aracın sesi; motor sesi, titreşimden kaynaklı sesler, rüzgâr sesleri gibi bazı seslerin bir araya gelmesiyle oluşur. Doğal olarak bu ses; araçta kullanılan yakıt türüne, motor çeşidine, yalıtım seviyesine, malzeme yapısına ve çeşitliliğine göre farklılık gösterir. Aynı marka, model ve özelliklere sahip araçlar, aynı şartlarda çalıştı̆̆ zaman yaklaşık olarak aynı sesleri üretmelerine (De Angelis ve diğ., 2016; Randall, 1987) rağmen bu sesler aracın kullanımına ve zamana bağlı olarak farklı parçalarının farklı seviyelerde yıpranmasından dolayı neredeyse benzersiz hale gelmektedir.

Bu çalışmada amacımız durağan halde bir aracın üretmiş olduğu bu benzersiz sesi "aracın sesli imzası" olarak tanımlayıp kaydetmektir. Bu sayede aracın sesli imzası kayıt edildikten sonra yeni bir ses algılandığında; bu sesin tanımlanması ve eğer daha önceden kayıtlı bir ses ise aracın sınıflandırılması için kullanılmasıdır. Geliştirilen bu sistemle günümüzde birçok uygulama mümkün olacaktır. Örneğin acil durumlarda, araç içerisinde telefon ile konuşan kişiden arka planda olan motor sesinin duyulması ile aracın belirlenmesi mümkün olabilecektir. Günümüzde üretilen araçların neredeyse tümü akıllı (Hahn ve diğ., 2019) ve sesli tanıma sistemleri için gerekli donanımsal alt yapıya sahiptir (Bisio ve diğ., 2018). Bu altyapı ile birlikte, tanımlanan "sesli imzalar" bütünleşik halde çalışabilecektir. Böylelikle araç çalıştırıldığı anda eğer aracın çalışma sesinin değişimine sebep olan bir arıza var ise bunun için öncü bir uyarı sistemi olacaktır.

\section{BENZER ÇALIŞMALAR (RELATED WORKS)}

2019 yılında yapılan bir çalışmada, düşük maliyetli, yapay sinir ağı ile çalışan, mikrodenetleyici tabanlı gömülü bir sistem geliştirmişlerdir. Geliştirdikleri sistem yol üzerinde herhangi bir uzaktan kontrol bağlantısına ihtiyaç duymadan yerleştirilip, geçen araçların motor seslerinden geçen araç olup/olmadığını algılamaktadır (Montino ve Pau, 2019). 2018 yılında yapılan başka bir çalışmada ise veri olarak hem araç sesi hem de araç görüntüsü kullanılmıştır. Araç sesleri dişarıdan ve araca yaklaşık 30m mesafeden kayıt edilmiştir. Hem araçları türlerine göre hem de motor sesinden yakıt tipine göre ayırmayı hedeflemişlerdir. Araç türlerini sınıflandırmada \%97,45'e kadar, motor tipini belirlemede ise benzinli araçlarda \%100, dizellerde ise \%92,7'ye kadar başarı sağlamışlardır (Wieczorkowska ve dĭğ., 2018). 2015 yılında yapılan bir çalışmada, araç dışından kaydedilen sesler veri olarak kullanılmış ve araç türü tahminini \%80 başarı ile yapmışlardır (Mayvan ve diğ., 2015). 2015 yılında yapılan diğer bir çalışmada ise yine yoldan alınan sesler ile araç türü tespit edilmeye çalışılmıştır. Çalışmalarında Extreme Learning Machine ile melezlenmiş özel bir Genetik Algoritma sınıfına (kısıtlı arama ile) dayalı yöntem kullanmışlardır. \%74,83 - \%93,74 arasında oranlarda başarılı sınıflandırma yapabilmişlerdir (Alexandre ve diğ., 2015). 2013 yılında yapılan bir çalışmada yaklaşmakta olan aracın sesinden araç türü ve uzaklığ1 tahmin edilmeye çalışılmıştır. Hareket halindeki araçların farklı çevrelerde üretmiş oldukları ses kaydedilmiş ve özbağınımlı model algoritma ile analiz edilmiştir. Bu sesin sınıflandırılması için PNN modelini kullanmışlardır. \%92,8 başarıyla araç türünü ve mesafesini tahmin edebilmişlerdir (Paulraj ve diğ., 2013). 2013 yılında yapılan diğer bir çalışmada, yola yerleştirmiş oldukları mikrofon ile yoldan geçen araçların seslerini kayıt edilmiş ve ANN/KNN algoritmalarını kullanarak sınıflandırılmıştır. Araç türünün tespitinde; KNN algoritmasında \%50,62 başarıya, ANN algoritmasında ise \%73,42 başarıya ulaşmışlardır (George ve diğ., 2013). 2013 yılında yapılan diğer bir çalışmada ise motor sesinden araç tanıma için ANN ve NBC algoritmalarının başarısını karşılaştırmıştır. Araç seslerini araç dışında kaydetmişlerdir. ANN için \%88,7 NBC için \%92,3 doğru sınıflandırma yapabilmişlerdir (Johnstone ve 
Woodward, 2013). 2009 da yapılan bir çalışmada, Matlab platformunda zaman kodlu sinyal işleme ve tanıma yöntemi (TESPAR) ile Archetypes tekniği birleştirilerek kullanılmıştır. Altı farklı araçtan kaydedilen 45 ses verisi üzerinde çalışmışlardır. Bazı araç modellerini belirlemede başarılı iken bazılarını ise yaklaşık \%50 hata ile sınıflandırmışlardır (Ghiurcau ve Rusu, 2009). 2007 yılında hazırlanan bir tez çalışmasında yol üzerinde araçlardan toplanan seslerden araç türü tespiti yapılmıştır. SVM algoritmasını kullanarak yapılan bu çalışmada \%87 'ye kadar doğru sınıflandırma yapabilmiştir. Ancak araç hızları veya trafik arttıkça bu başarı oranı oldukça düşmektedir (Erb, 2007). 1998 yılında Wu ve ark. yaptıkları bir çalışmada hareket halindeki araçların seslerini kullanılarak araç türünü tahmin etmeye çalışmışlardır. Ancak o zamanki teknoloji imkanlarının yoksunluğundan istemiş oldukları sonuçları elde edememişlerdir (Wu ve diğ., 1998). Ses sinyallerini kullanarak araç sınıflandırma araştırmacıların önem verdiği konulardan olup bununla ilgili Literatürde farklı algoritmalar geliştirerek farklı sınıflandırma sonuçları elde eden birçok çalışma (Dalir ve diğ., 2018; Paulraj ve diğ., 2013; Wieczorkowska ve diğ., 2018) olmuştur. Bu çalışmada sunulan yöntem ile bu çalışmaya konu olan benzer çalışmalar sonuç kısmında karşılaştırılmıştır.

\section{MATERYAL VE YÖNTEM (MATERIAL AND METHOD)}

\section{Veri Toplama (Data Collection)}

Çalışır durumda olan 22 aracın rölantideki araç içi sesi kaydedilmiştir. Ses kaydedilirken standart mikrofon kullanılmış ve aracın sürücü koltuğu ile yan yolcu koltuğunun ortasına konumlandırılmıştır. Araç sesleri kayıt edilirken ortam seslerinin olmamasına dikkat edilmiştir. Her bir ses kaydı en az 210 saniye olacak şekilde alınmıştır. Bu ses dosyaları iki saniyelik parçalara ayrıştırılmıştır. Böylece her araca ait 100'den fazla ses kaydı oluşturulmuştur. $44100 \mathrm{~Hz}$ örnekleme oranı ile toplam 2416 ses kaydı analiz edilmiştir. Çizelge 1'de araçlara yönelik detaylar verilmiştir. Sonrasında kaydedilen sesler Local Binary Pattern (LBP) yöntemi kullanılarak analiz edilmiştir.

Araçların yalnızca beş tanesi Benzin+LPG yakıt türüne sahip olup kalanların tümü dizel yakıtlı araçlardır. Araçların içerisinden dört tanesi aynı marka, aynı model, aynı yakıt türü ve aynı motor hacminde olup üretim yılı ve kilometre değerleri farklıdır. Böylece yapılacak tahminde hem tüm araçların birbirinden nasıl ayrıştığı hem de aynı özellikte olmalarına rağmen araçların ayrışıp ayrışmadı̆̆ı göstermek amaçlanmıştır.

\section{Yerel İkili Örüntü (LBP) (Local Binary Pattern)}

LBP, bir resmin her pikselini, o pikselin çevresindeki 3x3 alanda komşu olduğu pikseller ile eşleştiren ve sonuçta ikili sayı sisteminde tek bir değer üreterek bu pikselleri etiketleyen basit ancak çok etkili bir doku operatörüdür. Şekil-1a'da işaretli pikseli "merkez piksel" olarak ele alalım; eşlik tablosu çıkarabilmek ve LBP değerini elde edebilmek için komşu olduğu diğer sekiz piksel ile büyüklük/küçüklük ilişkisine bakılır. Eğer merkez pikselin değeri komşusu olan pikselden büyükse karşılaştırması yapılan pikselin konumuna 1, küçükse 0 yazılarak eşik tablosu hazırlanır. Çıkarılan tablodan sekiz bitlik LBP değeri ikilik sayı formatında elde edilebilir. Bunun için tablonun ilk hücresindeki değer en yüksek değerlikli bit olacak şekilde saat yönüne doğru ilerleyerek LBP ikili sayı değeri elde edilir. Kolay kullanım ve okuma için bu değer LBP kod olarak adlandırılarak onluk sayıya çevrilir ve ilgili piksel bu değer ile etiketlenir. 
Çizelge 1. Araç Detayları

Table 1. Details of the Vehicles

\begin{tabular}{|l|l|l|l|l|l|l|l|}
\hline ID & Marka & Model & \multicolumn{1}{|c|}{ Seri } & $\begin{array}{c}\text { Üretim } \\
\text { Y1li }\end{array}$ & $\begin{array}{l}\text { Motor } \\
\text { Hacmi }\end{array}$ & Yakıt Türü & KM \\
\hline 1 & Renault & Fluence & Icon & 2013 & 1500 & Dizel & 150000 \\
\hline 2 & Fiat & Egea & Urban & 2016 & 1300 & Dizel & 72000 \\
\hline 3 & Toyota & Corolla & Comfort Extra & 2012 & 1600 & Benzin+LPG & 84000 \\
\hline 4 & Wosvagen & Passat & Varyant & 2013 & 1600 & Dizel & 160000 \\
\hline 5 & Scoda & SuperB & Prestige & 2018 & 1600 & Dizel & 60000 \\
\hline 6 & Renault & Fluence & Icon & 2015 & 1500 & Dizel & 83000 \\
\hline 7 & Fiat & Linea & Active Plus & 2012 & 1300 & Dizel & 170000 \\
\hline 8 & Honda & Civic & Eco Elegans & 2012 & 1600 & Benzin+LPG & 104000 \\
\hline 9 & Renault & Fluence & Dynamic & 2011 & 1500 & Dizel & 220000 \\
\hline 10 & Peugeot & 301 & Allure & 2016 & 1600 & Dizel & 65000 \\
\hline 11 & Audi & A3 & Ambiante & 2014 & 1600 & Dizel & 78000 \\
\hline 12 & Fiat & Egea & Easy & 2017 & 1300 & Dizel & 86000 \\
\hline 13 & Fiat & Marea & Liberty & 2004 & 1600 & Benzin+LPG & 180000 \\
\hline 14 & Fiat & $500 x$ & Cross Plus & 2017 & 1600 & Dizel & 28000 \\
\hline 15 & Wosvagen & Polo & Comfort Line & 2015 & 1200 & Benzin+LPG & 20000 \\
\hline 16 & Mercedes & E200 & Elegance & 1995 & 2000 & Benzin+LPG & 270000 \\
\hline 17 & Scoda & Octavia & Elegance & 2014 & 1600 & Dizel & 69000 \\
\hline 18 & Wosvagen & Passat & R-Line & 2017 & 1600 & Dizel & 45000 \\
\hline 19 & Renault & Fluence & Extreme & 2015 & 1500 & Dizel & 141000 \\
\hline 20 & Chevrolet & Aveo & LTZ & 2012 & 1300 & Dizel & 148000 \\
\hline 21 & Ford & Focus & Trends & 2015 & 1600 & Dizel & 132000 \\
\hline 22 & Renault & Megane & Touch & 2017 & 1500 & Dizel & 94000 \\
\hline
\end{tabular}

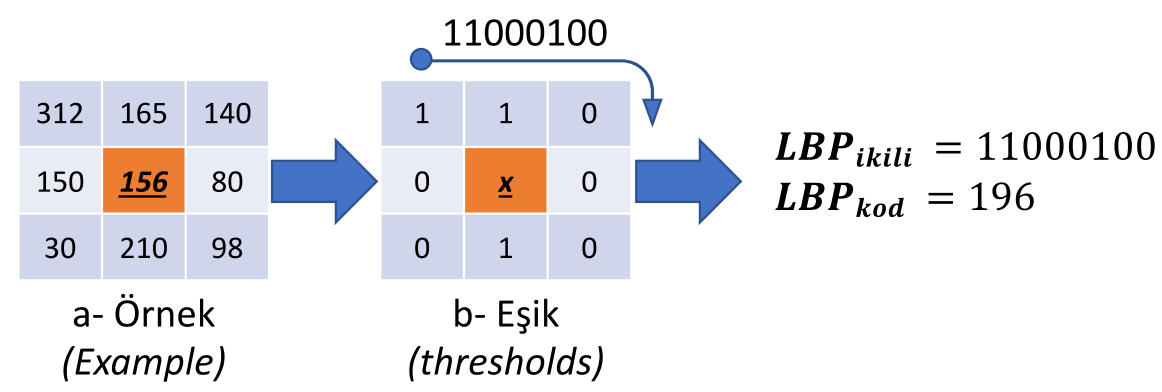

Şekil 1. Yerel İkili Örüntü (LBP) mantığ1

Figure 1. Logic of the Local Binary Pattern

LBP metodolojisi doku analizinde önemli ilerlemelere yol açmıştır. Araştırma ve uygulamalarda tüm dünyada yaygın olarak kullanılmaktadır (Pietikäinen, 2010). LBP'nin temel kullanım amacı yüz tanıma, doku tanıma, hareket analizi gibi (Da Costa ve diğ., 2019) görüntü işleme ve makine görmesine yönelik uygulamalardır (Huang ve diğ., 2011). Bunun en temel nedeni ise LBP'nin ayrıştırma da olan başarısı ve hesaplama kolaylığı olarak gösterilebilir. LBP'nin hesaplamalarda sağladığı basitlik ve ayrıştırma başarısı bu çalışmada görüntü işleme yerine ses sınıflandırma amacıyla kullanılmıştır. LBP görüntü işleme açısından oldukça etkili bir tekniktir ancak görüntü üzerindeki parazitlere karşı çok hassas olabilmektedir. Örneğin pikseller üzerine gelecek ufak bir aydınlık, piksel değerlerinin değişmesine bu sebeple LBP değeri hesaplarken kullanılacak 1'lerin 0 veya $0^{\prime}$ ların 1 olmasına sebep olabilir. Bir ses genliği (zaman-frekans gösterimi) iki boyutlu bir resim olarak düşünülerek, kaydedilen bir ses verisi genliği üzerinde LBP ile doku analizi yapmak mümkündür (Kobayashi ve Ye, 2017; Thwe ve War, 2017). 


\section{Kübik SVM (Cubic SVM)}

Destek Vektör Makinesi (Support Vector Machine - SVM) sınıflandırma tekniği düşük bellek alanı problemini aşmada kullanılan yararlı bir yöntemdir. SVM sınıfları mümkün olan en iyi şekilde ayırmak için çok boyutlu bir hiper düzlem bulur (Lin ve Wei, 2005). Kübik SVM sınıflandırıcısı sınıflandırıcının çekirdek fonksiyonunun kübik olduğu durumlarda ancak kullanılabilir. Aşağıdaki Denklem 1 ile kullanılır. Buradaki çalışmada Matlab programı içerisinde kübik SVM yönteminin varsayılan parametreleri kullanılmıştır.

$$
K\left(x_{i}, x j\right)=\left(x_{i}^{T} x j\right)^{3}
$$

\section{Bulgular (Findings)}

Çalışma içerisinde 22 tane aracın rölantide iken çıkartıkları araç içi sesleri kayıt altına alınmıştır. Ses dosyaları üçer saniyelik parçalara ayrılmış fakat üzerinde gürültü giderme benzeri hiçbir filtreleme ya da önişleme yapılmamıştır. LBP öznitelik çıkarma yöntemi Matlab platformunda ses dosyalarına uygulanarak 256 öznitelik çıkarılmıştır. Elde edilen veriler yine Matlab platformunda sınıflandırma algoritmaları kullanılarak analiz edilmiştir. 10-katlı çapraz doğrulama ile veriler eğitim ve test olarak ayrıştıılmıştır. Sınıflandırma algoritmalarının tümünde varsayılan parametreler kullanılmıştır. Öncelikle 22 aracın da içerisinde yer aldığı bir analiz yapılmış ve Cubic SVM algoritması ile \%95,2 oranında başarılı sınıflandırmalar yapılmıştır. Aşağıdaki Çizelge 2'de karışıklık matrisi gösterilmiştir.

Çizelge 2. Tüm Araçlar için Cubic SVM Algoritması ile Sonuçların Karışıklık Matrisi

Table 2. Confusion Matrix of Results with Cubic SVM Algorithm for All Vehicles

\begin{tabular}{|c|c|c|c|c|c|c|c|c|c|c|c|c|c|c|c|c|c|c|c|c|c|c|c|c|}
\hline & \multicolumn{22}{|c|}{ Tahmin Değerleri } & \multirow{2}{*}{ Tp. } \\
\hline & & 1 & 2 & 3 & 4 & 5 & 6 & 7 & 8 & 9 & 10 & 11 & 12 & 13 & 14 & 15 & 16 & 17 & 18 & 19 & 20 & 21 & 22 & \\
\hline \multirow{22}{*}{ 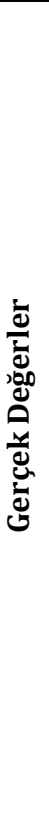 } & $\mathbf{1}$ & 100 & 0 & 0 & 0 & 0 & 3 & 0 & 2 & 1 & 0 & 0 & 0 & 1 & 0 & 0 & 0 & 0 & 0 & 0 & 0 & 0 & 0 & 107 \\
\hline & 2 & 0 & 102 & 0 & 0 & 0 & 0 & 0 & 0 & 0 & 0 & 0 & 2 & 3 & 0 & 0 & 0 & 0 & 0 & 0 & 0 & 0 & 0 & 107 \\
\hline & 3 & 1 & 0 & 98 & 0 & 0 & 0 & 0 & 2 & 0 & 0 & 0 & 0 & 4 & 0 & 0 & 0 & 0 & 0 & 0 & 0 & 0 & 0 & 105 \\
\hline & 4 & 0 & 0 & 3 & 103 & 0 & 0 & 0 & 0 & 0 & 0 & 0 & 0 & 0 & 0 & 0 & 0 & 0 & 0 & 0 & 0 & 0 & 0 & 106 \\
\hline & 5 & 0 & 0 & 0 & 0 & 108 & 0 & 1 & 0 & 1 & 0 & 1 & 0 & 0 & 0 & 0 & 0 & 0 & 0 & 0 & 0 & 0 & 0 & 111 \\
\hline & 6 & 0 & 0 & 0 & 0 & 0 & 98 & 0 & 0 & 3 & 0 & 0 & 0 & 0 & 0 & 0 & 0 & 0 & 0 & 0 & 0 & 0 & 0 & 101 \\
\hline & 7 & 0 & 0 & 0 & 0 & 1 & 0 & 104 & 0 & 0 & 1 & 0 & 0 & 0 & 0 & 0 & 0 & 0 & 0 & 0 & 0 & 0 & 0 & 106 \\
\hline & 8 & 0 & 0 & 0 & 0 & 2 & 5 & 0 & 94 & 0 & 2 & 0 & 0 & 3 & 0 & 0 & 0 & 0 & 0 & 0 & 0 & 0 & 0 & 106 \\
\hline & 9 & 4 & 0 & 0 & 0 & 0 & 0 & 1 & 0 & 101 & 0 & 0 & 0 & 0 & 0 & 0 & 0 & 0 & 0 & 0 & 0 & 0 & 0 & 106 \\
\hline & 10 & 0 & 0 & 0 & 0 & 0 & 0 & 2 & 0 & 0 & 104 & 0 & 0 & 0 & 0 & 0 & 0 & 0 & 0 & 0 & 0 & 0 & 0 & 106 \\
\hline & 11 & 0 & 0 & 0 & 0 & 4 & 0 & 0 & 0 & 1 & 0 & 101 & 0 & 1 & 0 & 0 & 0 & 0 & 0 & 0 & 0 & 0 & 0 & 107 \\
\hline & 12 & 0 & 5 & 0 & 0 & 1 & 0 & 0 & 0 & 0 & 0 & 0 & 98 & 2 & 0 & 0 & 0 & 0 & 0 & 0 & 0 & 0 & 0 & 106 \\
\hline & 13 & 1 & 0 & 0 & 0 & 0 & 0 & 0 & 14 & 0 & 0 & 0 & 0 & 91 & 0 & 0 & 0 & 0 & 0 & 0 & 0 & 0 & 0 & 106 \\
\hline & 14 & 0 & 3 & 0 & 0 & 2 & 0 & 0 & 0 & 0 & 0 & 0 & 0 & 0 & 104 & 0 & 0 & 0 & 0 & 0 & 0 & 0 & 0 & 109 \\
\hline & 15 & 0 & 1 & 1 & 0 & 0 & 0 & 0 & 0 & 0 & 0 & 0 & 0 & 0 & 0 & 104 & 0 & 0 & 0 & 0 & 0 & 0 & 0 & 106 \\
\hline & 16 & 0 & 0 & 0 & 0 & 0 & 0 & 0 & 0 & 0 & 0 & 0 & 0 & 0 & 0 & 0 & 114 & 0 & 0 & 0 & 0 & 7 & 0 & 121 \\
\hline & 17 & 0 & 0 & 0 & 0 & 0 & 0 & 0 & 0 & 0 & 0 & 0 & 0 & 0 & 0 & 0 & 6 & 105 & 0 & 0 & 0 & 2 & 0 & 113 \\
\hline & 18 & 0 & 0 & 0 & 0 & 0 & 0 & 0 & 0 & 0 & 0 & 0 & 0 & 0 & 0 & 0 & 0 & 0 & 112 & 0 & 0 & 1 & 0 & 113 \\
\hline & 19 & 0 & 0 & 0 & 0 & 0 & 0 & 0 & 0 & 0 & 0 & 0 & 0 & 0 & 0 & 0 & 1 & 0 & 0 & 114 & 1 & 0 & 0 & 116 \\
\hline & 20 & 0 & 0 & 0 & 0 & 0 & 0 & 0 & 0 & 0 & 0 & 0 & 0 & 0 & 0 & 0 & 0 & 0 & 0 & 1 & 110 & 0 & 0 & 111 \\
\hline & 21 & 0 & 0 & 0 & 0 & 0 & 0 & 0 & 0 & 0 & 0 & 0 & 0 & 0 & 0 & 0 & 4 & 0 & 0 & 1 & 0 & 131 & 1 & 137 \\
\hline & 22 & 0 & 0 & 0 & 0 & 0 & 0 & 0 & 0 & 0 & 0 & 0 & 0 & 0 & 0 & 0 & 1 & 0 & 0 & 0 & 1 & 0 & 108 & 110 \\
\hline
\end{tabular}

\section{SONUÇ VE TARTIŞMA (RESULTS AND DISCUSSION)}

22 aracın rölantideki araç içi sesi kaydedilip toplam 2416 ses kaydı analiz edilmiştir. Matlab platformunda LBP ile ses verilerinden 256 adet özniteliği çıkarılarak, Cubic SVM algoritması ile \%95,2 
oranında başarılı sınıflandırma yapılmıştır. Elde edilen bu yüksek başarı oranı her aracın aynı marka ve model bile olsalar kendilerine özgü bir "sesli imzalarının" olduğunu göstermektedir. Bu farklılığın özellikle aynı marka ve model araçlarda bile görülmesinin temel sebebi araçlarda kullanılan malzemelerin farklı zamanlarda ve farklı seviyelerde yıpranmasının yanı sıra araçlarda kullanılan yalıtım malzemelerinin, yakıtın, filtrelerin hatta motor yağı gibi küçük detayların bile etkili olduğu düşünülmektedir.

Literatürde yer edinmiş benzer çalışmalar ile sunulan çalışmanın arasındaki farklılıklar analiz edilerek Çizelge 3 'te sunulmuştur. Çizelge incelendiği zaman literatürde sunulan konu ile ilgili çalışmaların çoğunun araç türünü belirlemeye yönelik olduğu görülmektedir. Bunun yanı sıra farklı olarak ses kayıtları araç dışarısından alınmaktadır, bu sebeple alınan ses kayıtlarının içerisinde rüzgâr sesi, lastik sesi gibi birçok farklı etken sesler dahil olmaktadır. Sunulan çalışmada ise araç içerisinden alınan çok küçük bir ses parçası ile \%95,2 oranında araç doğru olarak tespit edilebilmiştir.

Çizelge 3. Benzer çalı̧̧malar ile karşılaştırma

Table 3. Comparison with similar studies

\begin{tabular}{|c|c|c|c|}
\hline $\begin{array}{c}\text { Benzer } \\
\text { Çalışmalar }\end{array}$ & Kullanılan yöntem & $\begin{array}{l}\text { Ulaşılan } \\
\text { Başarı }\end{array}$ & Sunulan çalışma ile farklılıkları \\
\hline $\begin{array}{l}\text { Montino ve } \\
\text { Pau, } 2019\end{array}$ & Yapay Sinir Ağ1 & $\% 97,5$ & $\begin{array}{l}\text { Ortam seslerini sınıflandırarak sadece geçen araç } \\
\text { olup/olamadığını algılamaktadır. Geçen araç için } \\
\text { herhangi bir tanımlayıcı bilgi vermemektedir. Sesler } \\
\text { araç dışında kaydedilmektedir. }\end{array}$ \\
\hline $\begin{array}{l}\text { Wieczorkowsk } \\
\text { a ve ark., } 2018\end{array}$ & $\begin{array}{l}\text { RF, DL, SVM(linear, } \\
\text { quadratic, RBF kernel) }\end{array}$ & $\% 97,45$ & $\begin{array}{l}\text { Sadece araç türü ve motor tipi tespit için } \\
\text { geliştirilmiştir. Araç marka, model ve serisini bulmaya } \\
\text { yönelik değildir. Ses araç dışında kayıt edilmektedir. }\end{array}$ \\
\hline $\begin{array}{l}\text { Mayvan ve } \\
\text { ark., } 2015\end{array}$ & QDA, SVM & $\% 80$ & $\begin{array}{l}\text { Sadece araç türünü tespit için geliştirilmiştir. Araç } \\
\text { marka, model ve serisini bulmaya yönelik değildir. } \\
\text { Araç dışında kayıt alınmıştır. }\end{array}$ \\
\hline $\begin{array}{l}\text { Alexandre ve } \\
\text { ark., } 2015\end{array}$ & $\begin{array}{l}\text { Extreme Learning Machine ve } \\
\text { Genetik Algoritma }\end{array}$ & $\% 93,74$ & $\begin{array}{l}\text { Sadece araç türünü tespit için geliştirilmiştir. Araç } \\
\text { marka, model ve serisini bulmaya yönelik değildir. } \\
\text { Araç dışında kayıt alınmıştır. }\end{array}$ \\
\hline $\begin{array}{l}\text { Paulraj ve ark., } \\
2013\end{array}$ & $\begin{array}{c}\text { Analiz için autoregressive, } \\
\text { sınıflandırma için PNN }\end{array}$ & $\% 92,8$ & $\begin{array}{l}\text { Sadece aracın türünü ve mesafesini tahmin etmeye } \\
\text { yöneliktir. Araç dışında kayıt alınmıştır. }\end{array}$ \\
\hline $\begin{array}{l}\text { George ve ark., } \\
2013\end{array}$ & ANN/KNN Algoritmaları & $\% 73,42$ & $\begin{array}{l}\text { Sadece aracın türünü bulmaya yöneliktir. Ses kayıtları } \\
\text { yol üzerinde yapılmaktadır. }\end{array}$ \\
\hline $\begin{array}{l}\text { Johnstone ve } \\
\text { ark., } 2013\end{array}$ & ANN/NBC Algoritmaları & $\begin{array}{c}\% 88,7 \text { ve } \\
\% 92,3\end{array}$ & $\begin{array}{l}\text { Kullanılan metot farklı olmasının yanı sıra her bir } \\
\text { eğitim seti için kullanılan ses kaydı } 26.3 \text { s sürmektedir. } \\
\text { Ayrıca sesler araç dışarısında kaydedilmektedir. }\end{array}$ \\
\hline $\begin{array}{l}\text { Ghiurcau ve } \\
\text { Rusu, } 2009\end{array}$ & $\begin{array}{l}\text { Matlab platformunda TESPAR } \\
\text { ile Archetypes tekniği }\end{array}$ & $\begin{array}{c}\% 45,5- \\
\% 100\end{array}$ & $\begin{array}{l}\text { Sadece } 6 \text { araç modeli üzerinde çalışılmıştır. Bazı } \\
\text { araçları \%55,55 hata ile sinıflandırmışlardır. }\end{array}$ \\
\hline Erb, 2007 & SVM Algoritması & $\% 80-\% 87$ & $\begin{array}{l}\text { Sadece araç türünü bulmaya yöneliktir. Ses kaydı araç } \\
\text { dışında yapılmaktadır. }\end{array}$ \\
\hline $\begin{array}{l}\text { Sunulan } \\
\text { Çalışma }\end{array}$ & Local Binary Pattern (LBP) & $\% 95,2$ & \\
\hline
\end{tabular}

Sonraki çalışma olarak araç hareket halinde iken bu çalışmada sunulan yöntem ile aracın sınıflandırılmasını sağlamaktır. Bunun için araç hareket halinde iken kaydedilen sesten gürültü olarak nitelendirilecek ve aracın hareketine bağlı meydana gelebilecek sürtünme sesi, rüzgâr sesi, yol sesi gibi diğer seslerin giderilmesi gerekmektedir. Ayrıca aynı marka, model ve özelliklere sahip araçlar, aynı şartlarda çalıştığı zaman yaklaşık olarak aynı sesleri üretmesi (De Angelis ve ark., 2016; Randall, 1987) durumu kullanılarak, araca özgün "sesli imza" durumu dikkate alınarak kaç kilometre yol yaptığı üzerinde çalışılabilir. Bu sayede kayıtlı olmayan bir aracın sesi algılandığında araç km verisi ile oynanıp oynanmadığı bulunulabilir. Eğitim verisi olarak kullanılacak, araç tanıma sesleri dikkatle seçilmeli, 
kaydedilmeli ve sınıflandırılmalıdır. Kayıt yapılırken ortamda bulunacak farklı seslerin dikkate alınmaması sonuçlarda ve analizlerde hataya neden olabilir.

\section{KAYNAKLAR (REFERENCES)}

Alexandre, E., Cuadra, L., Salcedo-Sanz, S., Pastor-Sánchez, A., Casanova-Mateo, C., 2015, “Hybridizing extreme learning machines and genetic algorithms to select acoustic features in vehicle classification applications", Neurocomputing, 152, 58-68.

Bisio, I., Garibotto, C., Grattarola, A., Lavagetto, F., Sciarrone, A., 2018, "Smart and robust speaker recognition for context-aware in-vehicle applications", IEEE Transactions on Vehicular Technology, 67(9), 8808-8821.

Chu, S., Narayanan, S., Kuo, C.C.J., 2008, "Environmental sound recognition using MP-based features," IEEE International Conference on Acoustics, Speech, and Signal Processing, pp. 1-4, 2008.

Cowling, M., Sitte, R., 2003, “Comparison of techniques for environmental sound recognition", Pattern Recognition Letters, vol. 24, pp. 2895-2907.

Da Costa, M.V.B., Couto, C.M.V., Couto, L.N., 2019 October, “Face Recognition Using LBP on an Image Transformation Based on Complex Network Degrees", 32nd SIBGRAPI Conference on Graphics, Patterns and Images (SIBGRAPI), pp. 163-169.

Dalir, A., Beheshti, A.A., Masoom, M.H., 2018, “Classification of vehicles based on audio signals using quadratic discriminant analysis and high energy feature vectors", arXiv, arXiv:1804.01212.

De Angelis, G., De Angelis, A., Pasku, V., Moschitta, A., Carbone, P., 2016 October, “A simple magnetic signature vehicles detection and classification system for smart cities", IEEE International Symposium on Systems Engineering (ISSE), pp. 1-6.

Dokur, Z., Ölmez, T., 2008, “Heart sound classification using wavelet transform and incremental selforganizing map", Digital Signal Processing, 18(6), 951-959.

Erb, S., 2007, "Classification of vehicles based on acoustic features”, Doctoral Dissertation Thesis, Graz University of Technology, Austria.

George, J., Cyril, A., Koshy, B. I., Mary, L., 2013, “Exploring sound signature for vehicle detection and classification using ANN", International Journal on Soft Computing, 4(2), 29.

George, J., Mary, L., Riyas, K. S., 2013 December, “Vehicle detection and classification from acoustic signal using ANN and KNN". International Conference on Control Communication and Computing (ICCC), pp. 436-439.

Ghiurcau, M.V., Rusu, C., 2009 July, "Vehicle sound classification. Application and low pass filtering influence", International Symposium on Signals, Circuits and Systems, pp. 1-4.

Hahn, D.A., Munir, A., Behzadan, V., 2019, "Security and Privacy Issues in Intelligent Transportation Systems: Classification and Challenges", IEEE Intelligent Transportation Systems Magazine.

Han, W., Coutinho, E., Ruan, H., Li, H., Schuller, B., Yu, X., Zhu, X., 2016, “Semi-supervised active learning for sound classification in hybrid learning environments", PloS one, 11(9).

Huang, D., Shan, C., Ardabilian, M., Wang, Y., Chen, L., 2011, “Local binary patterns and its application to facial image analysis: a survey", IEEE Transactions on Systems, Man, and Cybernetics, Part C (Applications and Reviews), 41(6), 765-781.

Huang, R., Hansen, J.H.L., 2006, "Advances in unsupervised audio classification and segmentation for the broadcast news and NGSW corpora," IEEE Transactions on Audio, Speech, and Language Processing, 14(3): pp. 907- 919.

Jarnicki, J., Mazurkiewicz, J., Maciejewski, H., 1998, "Mobile object recognition based on acoustic information", Proc. of the 24th Annual Conf. of the IEEE Industrial Society, vol. 3, pp. 1564-1569.

Johnstone, M.N., Woodward, A., 2013 December, "Automated detection of vehicles with machine learning", 11th Australian Information Security Management Conference, DOI: 10.4225/75/57b65924343cd. 
Kobayashi, T., Ye, J., 2014 May, "Acoustic feature extraction by statistics based local binary pattern for environmental sound classification", IEEE International Conference on Acoustics, Speech and Signal Processing (ICASSP), pp. 3052-3056.

Kubera, E., Wieczorkowska, A., Skrzypiec, K., 2015 October, “Audio-based hierarchic vehicle classification for intelligent transportation systems", In International Symposium on Methodologies for Intelligent Systems, pp. 343-352, Springer, Cham.

Lavner, Y., Ruinskiy, D., 2009, "A Decision-Tree-Based Algorithm for Speech/Music Classification and Segmentation," EURASIP Journal on Audio, Speech, and Music Processing, doi:10.1155/2009/239892

Lin, Y.L., Wei, G., 2005, "Speech emotion recognition based on HMM and SVM", International conference on machine learning and cybernetics, 8, pp. 4898-4901.

Mayvan, A.D., Beheshti, S.A., Masoom, M.H., 2015, “Classification of vehicles based on audio signals using quadratic discriminant analysis and high energy feature vectors", International Journal on Soft Computing, 6(1), 53.

Mielke, M., Brück, R., 2013 June, "Smartphone application for automatic classification of environmental sound", the 20th International Conference Mixed Design of Integrated Circuits and Systems-MIXDES, pp. 512-515.

Montino, P., Pau, D., 2019 September, “Environmental Intelligence for Embedded Real-time Traffic Sound Classification", IEEE 5th International forum on Research and Technology for Society and Industry (RTSI), pp. 45-50.

Paulraj, M.P., Adom, A.H., Sundararaj, S., Rahim, N.B.A., 2013, “Moving vehicle recognition and classification based on time domain approach", Procedia Engineering, 53, 405-410.

Pietikäinen, M., 2010, “Local Binary Patterns", Scholarpedia, 5(3), 9775.

Radhakrishnan, R., Divakaran, A., Smaragdis, P., 2005, "Audio analysis for surveillance applications", IEEE WASPAA'05, pp. 158-161.

Randall, R.B., 1987, “Frequency analysis", California Üniversitesi, Bruel ve Kjaer, ISBN: 87-87355-07-8.

Salamon, J., Bello, J.P., 2015 April, “Unsupervised feature learning for urban sound classification”, IEEE International Conference on Acoustics, Speech and Signal Processing (ICASSP), pp. 171-175.

Salamon, J., Bello, J.P., 2017, “Deep convolutional neural networks and data augmentation for environmental sound classification", IEEE Signal Processing Letters, 24(3), 279-283.

Salamon, J., Jacoby, C., Bello, J. P., 2014 November, "A dataset and taxonomy for urban sound research", the 22nd ACM international conference on Multimedia, pp. 1041-1044.

Scheirer, E., Slaney, M., 1997, "Construction and evaluation of a robust multifeature speech/music discriminator," IEEE International Conference on Acoustics, Speech, and Signal Processing, pp. 13311334.

Tan, Y., Xu, Y., Das, S., Chaudhry, A., 2018 October, "Vehicle Detection and Classification in Aerial Imagery", 25th IEEE International Conference on Image Processing (ICIP), pp. 86-90.

Thwe, K.Z., War, N., 2017 June, "Environmental sound classification based on time-frequency representation", 18th IEEE/ACIS International Conference on Software Engineering, Artificial Intelligence, Networking and Parallel/Distributed Computing (SNPD), pp. 251-255.

Wieczorkowska, A., Kubera, E., Słowik, T., Skrzypiec, K., 2018, Spectral features for audio based vehicle and engine classification, Journal of Intelligent Information Systems, 50(2), 265-290.

Wu, H., Siegel, M., Khosla, P., 1998 May, "Vehicle sound signature recognition by frequency vector principal component analysis", IEEE Instrumentation and Measurement Technology Conference, vol. 1, pp. 429-434.

Yoo, I.-C., Yook, D., 2008, "Automatic sound recognition for the hearing impaired", IEEE Trans. on Consumer Electronic, vol. 54, pp. 2029- 2036.

Zhang, T., Kuo, C.C.J., 2001, "Audio content analysis for online audiovisual data segmentation and classification", IEEE Transactions on Speech and Audio Processing, 9(4): pp. 441 - 457. 